\title{
Polymeric photonic quasicrystal: Octonacci sequence and elasto-optic effect
}

\author{
Chittaranjan Nayak ${ }^{\mathrm{a}, *}$, Shuvendu Jena ${ }^{\mathrm{b}}$, Satyam Rout ${ }^{\mathrm{a}}$, Bhuvneshwer Suthar ${ }^{\mathrm{c}}$, Dinesh V. Udupa ${ }^{\mathrm{b}}$ \\ ${ }^{a}$ Department of Electronics and Communication Engineering, College of Engineering and Technology, SRM Institute of Science and \\ Technology, SRM Nagar, Kattankulathur, 603203, Kanchipuram, Chennai, TN, India \\ ${ }^{b}$ Atomic and Molecular Physics Division, Bhabha Atomic Research Centre, Mumbai, 400 085, India \\ ${ }^{c}$ Department of Physics, M.L.B. Govt. College, Nokha, 334803, Bikaner, Rajasthan, India
}

\begin{abstract}
Here we would like to discuss the light transmission modulation by the one-dimensional polymeric quasi-multilayer which is formed according to substitutional generalized Octonacci with PMMA and PS as the constituent materials. In particular, we will present some theoretical findings using the well-known transfer matrix method. PBG inter-band spacing and depth can be managed by choosing the appropriate generation number. The number of PBGs is the same while increasing in the generation number and are shifted symmetrically towards the designed frequency. It also reveals the aroused forbidden frequency band can be manipulated by changing the applied hydrostatic pressure and the thickness of the constituent polymeric materials. The increase in pressure shows a blue shift in the PBGs while the increase in thickness of the polymeric material provides a redshift to PBGs. The proposed structure could be another possible system for optical device design specially multi-band tunable optical reflectors.
\end{abstract}

Keywords: Elasto-optic effect, Octonacci sequence, transmission dynamics.

\section{Introduction}

Photonic crystal $(\mathrm{PhC})$ is an artificially engineered, periodic structure with the dielectric constants periodically varying in space. Yablonovitch [1] and John [2] independently proposed the concept of such superstructure in 1987, which affect the propagation of photons analogously to electrons in a crystal and this may result in the appearance of what is being called a photonic bandgap (PBG) 3. 4. The PBG of PhC suffers from being sensitive to the lattice structure for instant breaking the periodicity of the structure generates allowed states that are strongly localized around the defect in the PBG. Whilst modulation following a deterministic generation rule gives rise to a photonic quasicrystal (PQC) [5], is may practice for the same. Besides, the similar functionalities as periodic counterparts, such as PBG, negative refraction, the transmission properties of electromagnetic waves (EM) waves in PQC give some extraordinary results [6] like their branching band structures [7. The more widely studied PQC are the Fibonacci [8, 9, Thue-Morse 8, Cantor 10, Doubleperiod 8 and Rudin Shapiro [11. However, research in Octonacci PQC [12] has been so far mainly limited to theoretical investigations of multilayered structures. This sequence has a geometric origin: the octagonal AmmannBecker tiling is ruled by the Octonacci sequence.

A tunable $\mathrm{PhC}$ or PQC in which the PBG as well as the defect-mode can be tuned as desired by controlling

\footnotetext{
* Corresponding author:

Email address: 83chittaranjan@gmail.com (Chittaranjan Nayak)
}

parameters such as the refractive index [13. Therefore, the use of constituent building blocks with relatively more complex dielectric constant than simply losslessness can provide extra degree of freedom in the design of tunable $\mathrm{PhCs}$ and PQCs. In this line, the resent focus on those $\mathrm{PhCs}$ and PQCs whose dielectric properties can be controlled by the external influences such as magnetic field, electric field, temperature, pressure due to magneto-optic effect [14, 15, electro-optic effect [16, thermo-optic effect [17 and elasto-optic effect [18, 19] respectively. The elasooptic phenomena is well observed in transparent polymers such as polyamide-imide films (PAI), polyvinyl butyral. (PVB) polystyrene (PS) and poly methyl methacrylate (PMMA) and therefore is well accepted for practicing photonic structures. In this regards, polymer-based PCs and PQCs are an interesting alternative that can be used at very high frequency bands [19] particularly in the $\mathrm{THz}$ or mid-infrared $(10-100 \mathrm{THz})$ bands and has employed in very advanced applications such as infrared free space communications, biomedical, and environmental sciences, safety monitoring and many more.

In the present work, we explore the transmission spectra and the possibility new of PBGs from the Octonacci quasicrystal [12] which is composed of PMMA and PS [19]. The dielectric constant of such polymer can be tuned by the applied hydrostatic pressure. First, we present the structures under investigation and describe shortly the computational method we used in Sec. II. Our results about the transmittance spectra of light as well a detailed discussion over them are in Sec. III. Final conclusions are 


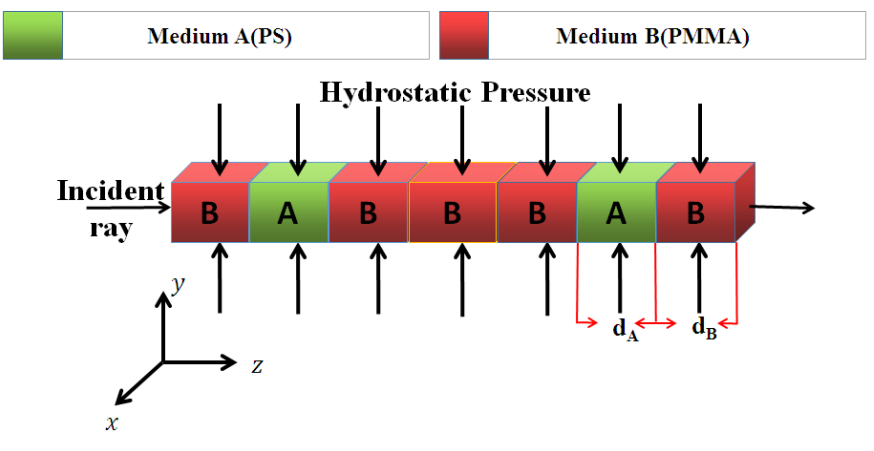

Figure 1: Colour line: Schematic illustration of the proposed Polymeric Octonacci photonic quasicrystal having generation 0number, $N=4$

given in Sec. IV.

\section{Methodology}

The geometrical PQC structure in this paper consists of a one dimensional Octonacci quasicrystal in an air environment, as illustrated in Fig.1. Layer $A$, with thickness $d_{A}$ (quarter wave condition), is fulfilled by PMMA, and is characterized by a hydrostatic pressure dependent refractive index $n_{A}$. Layer $B$, with thickness $d_{B}$ (quarter wave condition), is fulfilled by the PS, and is characterized by a hydrostatic pressure dependent refractive index $n_{B}$ [19]. The Octonacci sequence is created by the recurrence relation [12] $O_{n}=\left\{O_{n-1} O_{n-2} O_{n-1}\right\}$ for the generation number, $n \geq 3$, with initial condition: $O_{1}=\{A\}, O_{2}=\{B\}$. For the theoretical method employed for the transmission simulations of such structure we refer to Ref. 20]. Briefly, the characteristic matrix corresponding to the $i^{t h}$ layer is

$$
M[i]=\left[\begin{array}{ll}
m_{11} & m_{12} \\
m_{21} & m_{22}
\end{array}\right]
$$

and the characteristic matrix of the quasi sequence is

$$
M[T]=\left[\begin{array}{ll}
M_{11} & M_{12} \\
M_{21} & M_{22}
\end{array}\right]=\prod_{i=1}^{N} M[i]
$$

where, $N$ is the number of layer to required to construct the sequence and can be calculated by the recurrence relation, $N_{n}=2 N_{n-1}+N_{n-2}$ for $n \geq 3$, where $N_{1}=1$ and $N_{2}=1$. Finally, the transmittance, $T$, of the quasicrystal is calculated through the transmission coefficient which is the amplitude of the right propagating wave in the substrate region.

\section{Result and Discussions}

We now intend to investigate the transmittance spectra in generalized Octonacci one-dimensional PQC by using the calculations of the previous section. The considered PQCs are stacked of two types of layers namely, $A$ (PS) and $B$ (PMMA) [19] according to the substitution rules of

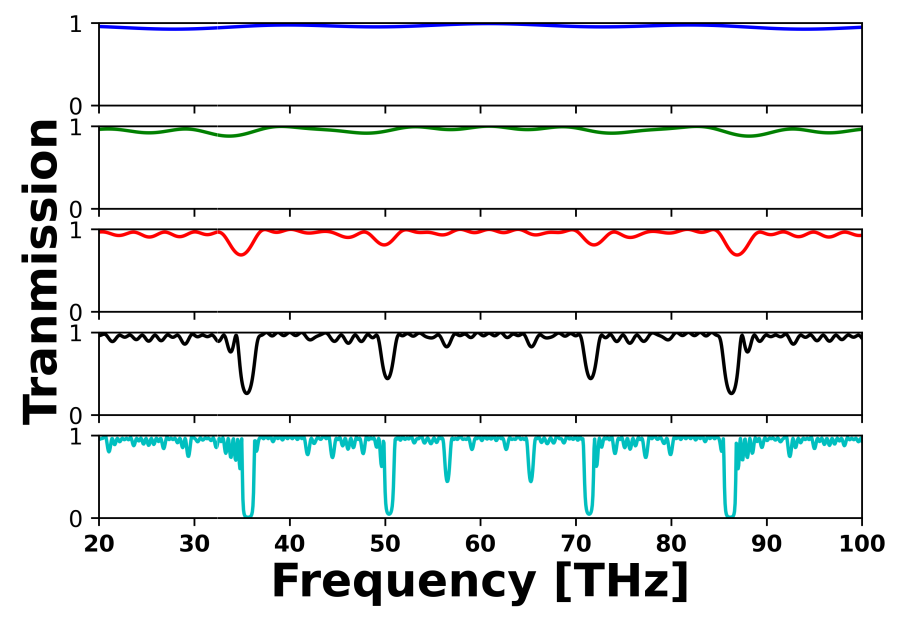

Figure 2: Colour line: Transmittance spectra as a function of incidence frequency $[\mathrm{THz}]$ for an Octonacci photonic quasicrystal contain two alternating polymer materials, PMMA and PS. Here, different sequence, $O_{n}$ having generation number, $n$ are presented. $n=4$ (blue), $n=5$ (green), $n=6$ (red), $n=7$ (black) and $n=8$ (cyan).

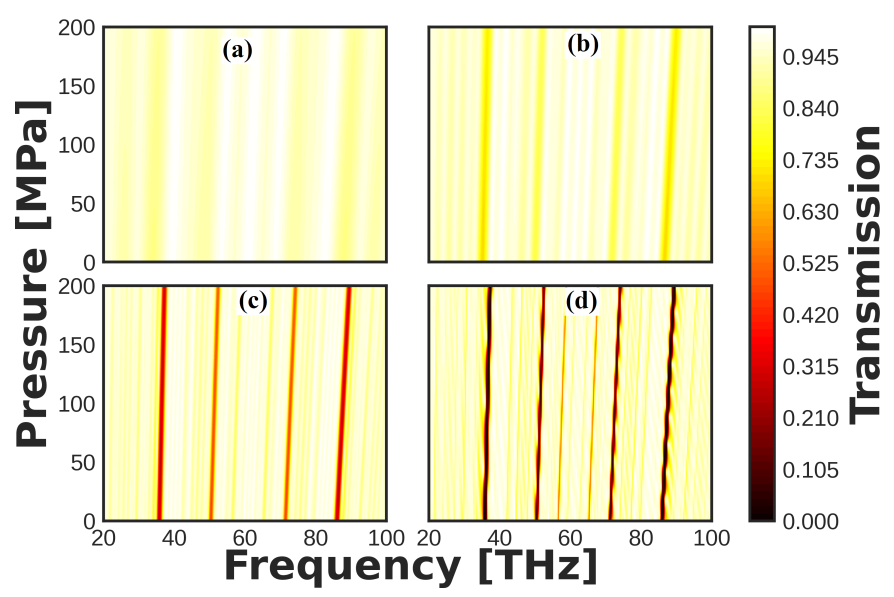

Figure 3: transmittance $\mathrm{T}$ in Colour map as a function of incidence frequency $[\mathrm{THz}]$ and hydrostatic pressure $[\mathrm{MPa}]$ for the polymeric Octonacci photonic quasicrystal having generation number, $n=5$ (a), $n=6$ (b), $n=7$ (c) and $n=8$ (d).

the Octonacci sequences [12. We compute the transmission properties of light incident normally through the air on the considered multi-layer structures. It is investigated under the influence of the parameters of the constituent materials such as the thickness of the layers and the applied external hydrostatic pressure. The pressure dependant refractive index of PS and PMMA is taken from Ref. [19. The structure is designed at $61.25 \mathrm{THz}$ which correspond to $d_{A}=780 \mathrm{~nm}$ and $d_{B}=830 \mathrm{~nm}$. The observation frequency range is fixed from 20 to $100 \mathrm{THz}$.

Fig. 2 illustrates the transmittance of the Octonacci multilayer, $S_{n}$ at generation number, $n=4$ (blue), 5 (green), 6 (red), 7 (black) and 8 (cyan) versus the frequency. For the Octonacci multilayer, $n=4$ (blue), oscillation in transmission with four dips at unit transmittance is noted. Such behaviour is aroused because of the spa- 
(a)

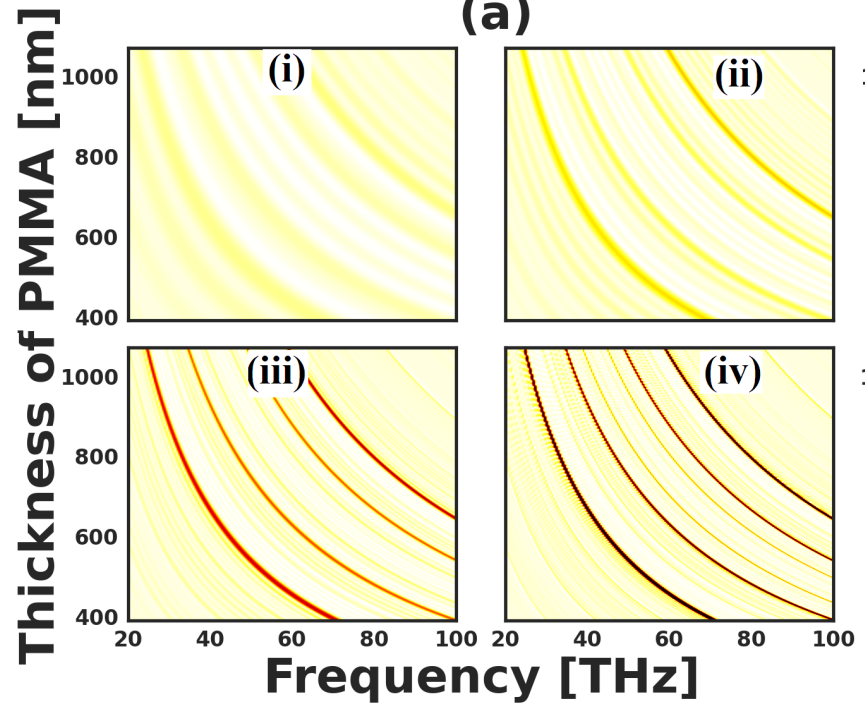

(b)

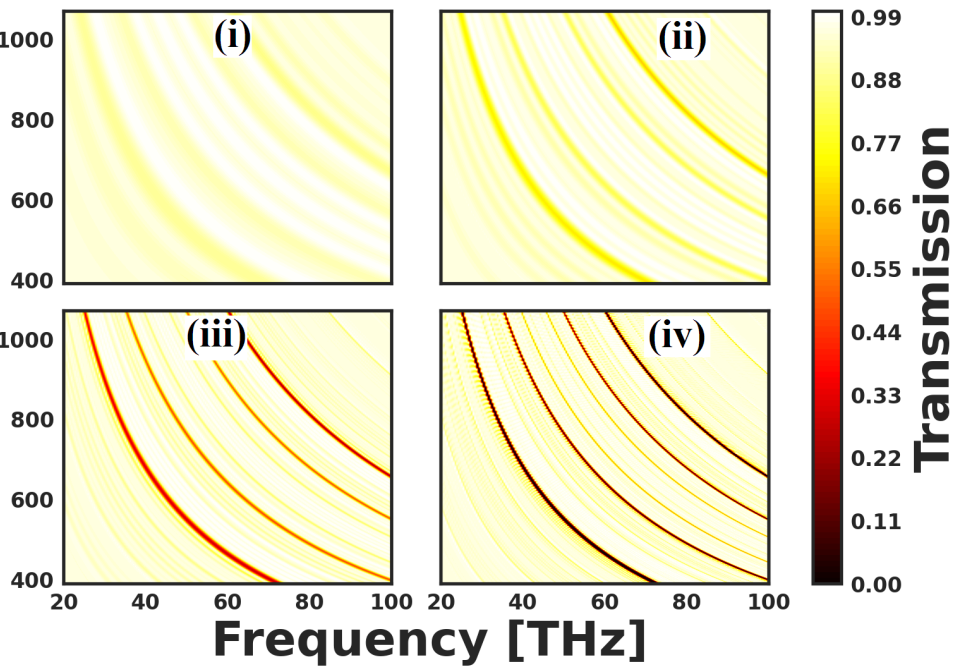

Figure 4: transmittance $\mathrm{T}$ in Colour map as a function of incidence frequency [THz] and thickness of the PMMA layer [nm] for the polymeric Octonacci photonic quasicrystal having generation number, $n=5$ (i), $n=6$ (ii), $n=7$ (iii) and $n=8$ (iv) both in panel (a) and (b). In panel (a) $P=0 \mathrm{MPa}$ whereas $P=100 \mathrm{MPa}$ in panel (b). Thickness of the PS is kept constant to $830 \mathrm{~nm}$ in both of the panels

tially variation in refractive index of two optically transparent material. At $n=5$ (green) these dips are intensified with some additional dips. In addition, these dips are symmetrically shift toward the designed frequency i.e. $61.25 \mathrm{THz}$. With increase in $n$ these four dips transformed to PBGs in the direction of propagation (Z-axis). Fig 3 presents the numerical results of increasing the hydrostatic pressure from 0 to $200 \mathrm{MPa}$ on the same sequences, expect the $n=4$, represents at the Fig 2. In detail, The Octonacci multilayer, $n=5$ at 'a', 6 at 'b', 7 at 'c' and 8 at 'd', respectively. In all the case, it was found that, increase in external hydrostatic pressure, the refractive index contrast decreases [19], causing the the PBG to blue shifted which is gives and opportunity for tunable multiband reflector design.

To realize the effect on the forbidden region introduced by the variation of the layer thicknesses of the polymeric Octonacci quasiperiodic stuctures, we consider the same sequences employed for Fig 3, Octonacci multilayer, $O_{n}$ at generation number, $n=5,6,7$ and 8 at hydrostatic pressure, $P=0 \mathrm{MPa}$ and at a fixed hydrostatic pressure, $P=100 \mathrm{MPa}$. At first we start our analysis to examine the effect of thickness on transmittance spectrum with respect to the changes in PMMA layer from $390 \mathrm{~nm}$ (one by eighth wave condition) to $1070 \mathrm{~nm}$ (half-wave condition) whereas the thickness of PS is kept constant to $830 \mathrm{~nm}$ (quarter-wave condition). The transmission spectrum for Octonacci multilayer, without any hydrostatic pressure, $P=0 \mathrm{MPa}, O_{n}$ at generation number, $n=5,6,7$ and 8 are depicted in Fig. 4 (a). Whereas in Fig. 4 (b) the same for $P=100 \mathrm{MPa}$ are depicted. From Fig. 4 (a), we observe that the aroused PBGs experiences a red shift and becomes narrow as the PMMA layers thickness increases. Moreover, the frequency spacing between also decreases with increase in it. While observing the effect of hydrostatic pressure in Fig. 4 (b), where hydrostatic pressure is equal to $100 \mathrm{MPa}$, a similar behaviour of the transmittance spectra is observed. Whereas the presence of hydrostatic pressure shift the PBGs to blue side, alike we shown in Fig. 3. These appeared modifications in the position and width of the forbidden regions aroused because of intrinsic consequences of each constituent elements.

In order to realize the effect on the forbidden region introduced by the variation of the layer thicknesses more clearly, we examine how the forbidden regions can be controlled with respect to the changes in PS layer thickness from $415 \mathrm{~nm}$ (one by eighth wave condition) to $1245 \mathrm{~nm}$ (half-wave condition) whereas the thickness of $d_{A}$ is kept constant to $780 \mathrm{~nm}$ (quarter-wave condition). The transmission spectrum for Octonacci multilayer, without any hydrostatic pressure, $P=0 \mathrm{MPa}, O_{n}$ at generation number, $n=5,6,7$ and 8 are depicted in Fig. 5 (a). Whereas in Fig. 5 (b) the same for $P=100 \mathrm{MPa}$ are depicted. From Fig 5, a very close response to the changes in PMMA layer thickness is observed. These alike appeared modifications in the position and width of the forbidden regions aroused because of the chose the polymeric material with very close dielectric constant to design the stack.

Then, we investigate the PQC where the addition of PMMA and PS is taken as constant. Here, the total thikness to form a period is $1610 \mathrm{~nm}$. Fig. 6 displays the transmittance spectra with increase of PMMA layer thickness from $390 \mathrm{~nm}$ (one by eighth wave condition) to $1070 \mathrm{~nm}$ (half-wave condition) whereas the thickness of PS layer is calculated with reference to PMMA layer thickness. The transmission spectrum for Octonacci multilayer, without any hydrostatic pressure, $P=0 \mathrm{MPa}, O_{n}$ at generation number, $n=5,6,7$ and 8 are depicted in Fig. 6 (a). 
(a)

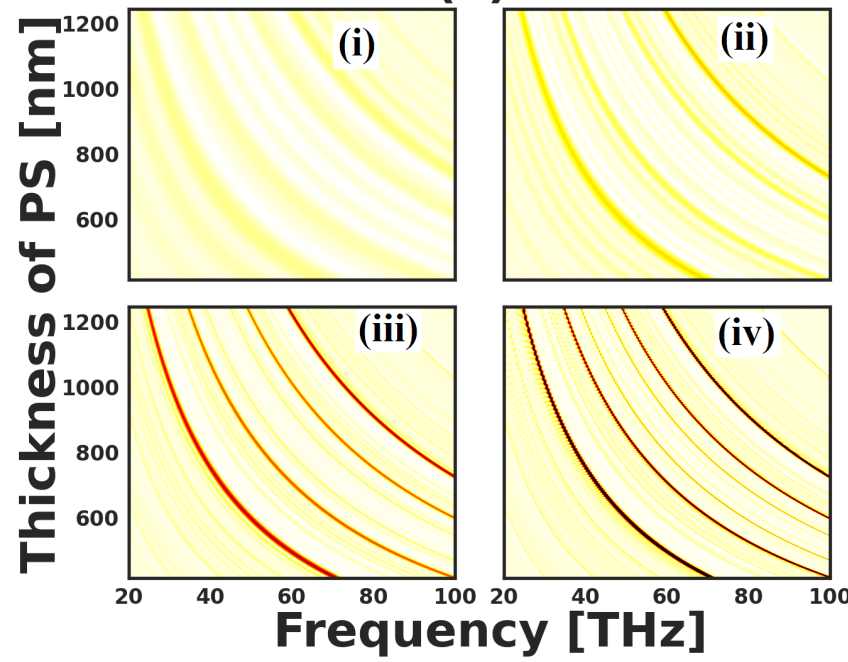

(b)

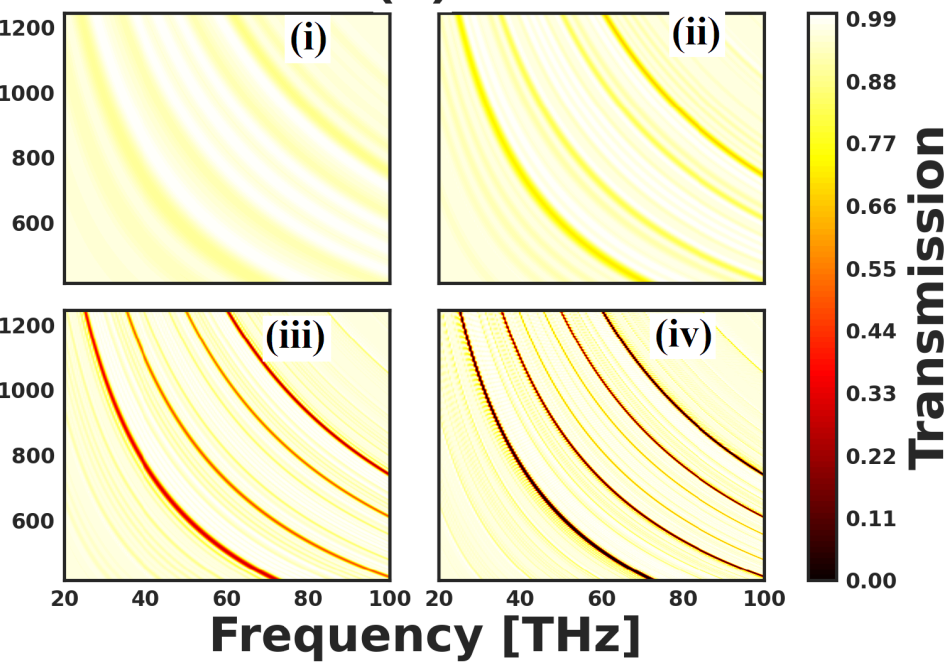

Figure 5: transmittance $\mathrm{T}$ in Colour map as a function of incidence frequency [THz] and thickness of the PS layer [nm] for the polymeric Octonacci photonic quasicrystal having generation number, $n=5$ (i), $n=6$ (ii), $n=7$ (iii) and $n=8$ (iv) both in panel (a) and (b). In panel (a) $P=0 \mathrm{MPa}$ whereas $P=100 \mathrm{MPa}$ in panel (b).Thickness of the PMMA is kept constant to $780 \mathrm{~nm}$ in both of the panels
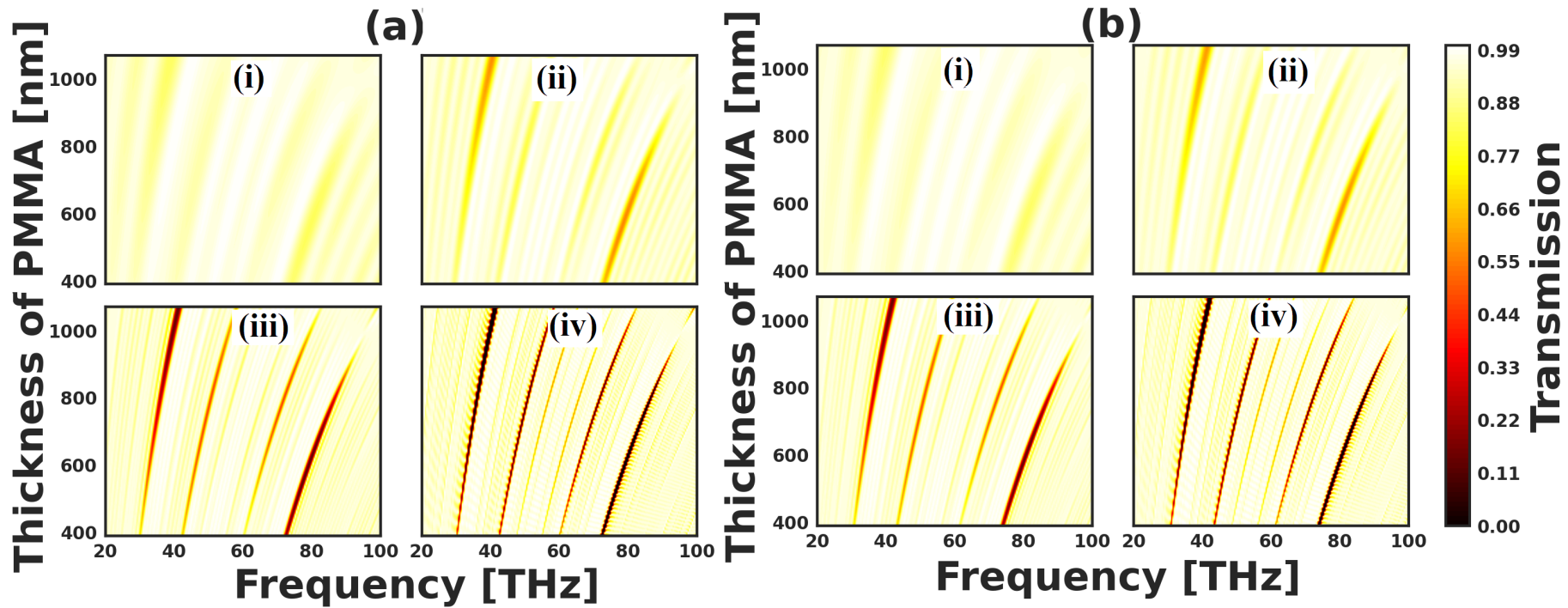

Figure 6: transmittance $\mathrm{T}$ in Colour map as a function of incidence frequency [THz] and thickness of the PMMA layer [nm] for the polymeric Octonacci photonic quasicrystal having generation number, $n=5$ (i), $n=6$ (ii), $n=7$ (iii) and $n=8$ (iv) both in panel (a) and (b). In panel (a) $P=0 \mathrm{MPa}$ whereas $P=100 \mathrm{MPa}$ in panel (b).Here, the sum of thickness of the PMMA and PS is always kept constant to 1610 nm in both of the panels

Whereas in Fig. 6 (b) the same for $P=100 \mathrm{MPa}$ are depicted. It can be found from the results that the PBGs within the frequency region are shifted to higher frequency when PMMA layer thickness increases.

In this case, the thickness of PMMA layer increases while the thickness of PS layer decreases correspondingly. Thererfore, the variation in PBG show in Fig. 6 is the combined of the increase in the thickness of PMMA and decrease in the thickness of PS layer. As previous study reflects that the increase in thickness of any layer (PMMA or PS) causes the shift in PBG towards lower frequency. Therefore, the decrease in the thickness of PS layer causes the shift towards higher frequency. From Fig. 6, it is clear that the overall the PBG shifted towards higher frequency suggests, the variation in PBG is more effective in case of reduction in the thickness of any layer compared with the increase in the thickness.

While the changes in the bandwidth of the PBGs are quite interesting, they are quite changes of PBGs in trend to our previous two cases, variation of PMMA as well as PS thickness. Moreover, the slope of PBGs are found to be more as from simple variation of PMMA and PS layer thickness. Therefore, in accordance with the application, the edge of the PBGs and their width can be effectively tuned by altering the thickness both of the layers is suggested. 


\section{Conclusions}

The transmittance of one dimensional ploymeric Octonacci structure is investigated with the change of applied hydrostatic pressure and the layer thickness of the constituent materials using the characteristic matrix. By comparing the transmittance spectra of different generation number $n$, we try to highlight the importance of PBG formation in PQC. We found that, initially, at $n=4$, the Octonacci distribution introduce four transmission dips in the computed frequency band and these are transformed to PBGs as the generation number increases. The formed PBGs in the transmission spectra of the PQC changes for different applied hydrostatic pressure and shifted to right with increase in it. The increase in thickness of the structure is imposed a mixed response on the aroused PBGs. More clearly, with increase in both PMMA and PS layer thickness the PBS are shifted to lower frequency and becomes very very marginally wider. On the other hand, the PBGs are shifted to higher frequency with increase in PMMA layer thickness while the addition of PMMA and PS layer thickness is always kept constant to $1610 \mathrm{~nm}$.

\section{Acknowledgements}

The author acknowledges the HOD, Department of Electronics and Communication Engineering, the Dean College of Engineering and Technology, and the Vice-Chancellor, SRM Institute of Science and Technology, Chennai, for their continuous encouragement.

\section{References}

[1] E. Yablonovitch, Phys. Rev. Lett. 58 (1987) 2059.

[2] S. Jhon, Phys. Rev. Lett. 58 (1987) 2486.

[3] C. M. Soukoulis, Photonic crystals and light localization in the 21st century, Springer Science \& Business Media, (2012).

[4] D. W. Prather, A. Sharkawy, S. Shi, J. Murakowski, G. Schneider, Photonic Crystals: Theory, Applications and Fabrication, Wiley Online Library, (2009).

[5] D. Shechtman, I. Blech, D. Gratias, Phys. Rev. Lett. 53 (1984) 1951.

[6] K. Mnaymneh, R. C. Gauthier, Opt. Express 47 (2007) 50895099.

[7] Z. V. Vardeny, A. Nahata, A. Agrawal, Nat. Photonics 7 (2013) 177-187.

[8] C. Nayak, C. H. Costa, A. Aghajamali, IEEE Trans. Plasma Sci. 47 (2019) 1726.

[9] A. Padhy, R. Bandyopadhyay, C. H. Costa, C. G. Bezerra, C. Nayak, Opt. Soc. Am. B 37 (2020) 3801-3808.

[10] N. B. Ali, Nano Commun. Netw. 13 (2017) 32-42.

[11] H. Rahimi, Opt. Mater. 57 (2015) 264-271.

[12] C. Nayak, A. Aghajamali, T. Alamfard, A. Saha, Physica B 525 (2017) 41.

[13] Q. Gong, X. Hu, Photonic crystals: principles and applications, CRC press, (2014).

[14] C. Nayak, Opt. Mater. 100 (2020) 109653.

[15] A. Rashidi, C. Nayak, C. G. Bezerra, C. H. Costa, F. A. Pinheiro, Appl. Opt. 87 (2005) 121110.

[16] M. Schmidt, U. Huebner, R. Boucher, Appl. Phys. Lett. 87 (2020) 11034-11040.
[17] C. Nayak, A. Aghajamali, M. Solaimani, J. K. Rakshit, D. Panigrahy, K. V. P. Kumar, B. Ramakrishna, Optik 222 (2020) 165290.

[18] F. Segovia-Chavesa, H. Vinck-Posadaa, Optik 196 (2019) 163141 .

[19] S. Jena, R.B. Tokas, S. Thakur, D.V. Udupa, . Physica E 114 (2019) 113627.

[20] M. Born, E. Wolf, Principles of Optics, Cambridge University Press, Cambridge, (2013). 